\title{
Investigating Agent-based Mobile Service Applications Integration with Reliable Wireless Communications
}

\author{
Sameh Abdel-Naby, Anara Sandygulova, Olga Zlydareva and Gregory M.P. O'Hare \\ CLARITY: Centre for Sensor Web Technologies \\ University College Dublin \\ Dublin, Ireland. \\ \{sameh, anara.sandygulova, olga.zlydareva, gregory.ohare\}@ucd.ie
}

\begin{abstract}
In this paper we investigate how recent advances in wireless communications can be integrated with agent-base service applications so that the connectivity between different users of the same environment, or remotely connected ones, is improved. We present two agent-based mobile service applications that employ software agents to represent end-users. We show how these applications are providing essential services for users on-the-run through distributed Bluetooth access points. We then briefly analyze possible future integration once advanced wireless communication is added to one of these services.
\end{abstract}

\section{INTRODUCTION}

Pocket Computing Devices (PCDs) devices such as Smartphones and PDAs are increasingly showing the necessity to obtain them and the reliability of using them. PCDs are part of the communications revolution which made it possible to virtually carry our offices anywhere we go. People now are using their PCDs to check their emails, exchange faxes, surf the Internet, edit documents and do shopping.

Recent integration of reliable telecommunication technologies such as EDGE, UMTS, HSDPA, or 802.15 and 802.16 are increasingly enabling a various number of heavyweight end-user service applications. Each of these applications is aimed at different type of service. For example, BarterCell is an agent-based application which provide the possibility for end-users to exchange similar value objects. ANDIAMO [1] is another agent-based application that enables users of pocket computing devices to do carpooling.

Our research effort addresses the possible integration of recent communication technologies in our daily life service applications that are agent-based. Many of these technologies are already used to provide services for users on the go, however, it is hardly happening that these technologies are also used in close networks for location-based services. Our role comes to motivate the idea of using recent communication technologies to increase the reliability of location-based services.

This paper is structured as follows. Sections II and III introduce briefly the BarterCell and ANDIAMO mobile service applications. Section IV explores the deployment of wireless communications into these agent-based mobile applications. Section V concludes our paper and presents our future work.

\section{BARTERCEll Platform Description}

The architecture of BarterCell, as shown in figure 1, relies on the user's capable pocket devices or PC to accomplish a

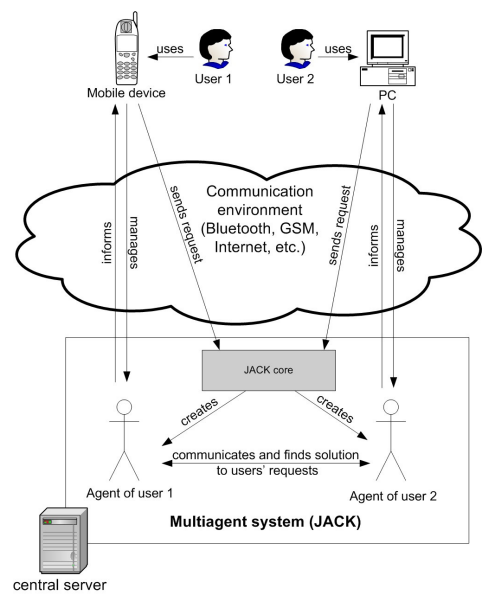

Fig. 1. The BarterCell Architecture

successful bartering task. Via the preinstalled Java application, users start by creating their own profiles using simple and user-friendly interface, insert their preferences, and add details related to the kind of items they are exchanging. If a PC is used, the user will be asked to directly upload the saved data to a central server which, in return, make it available to agents running on Jack [2].

Our system was deployed using distributed Bluetooth access points that were all located within a specific environment (e.g., university). Due to some technology limitations, users are asked to be present within the coverage of any connecting spot in order to transmit their data files to the central server. Regardless of the methods used to transmit the data, the processing and sequence of system instructions from this point on will be the same.

Once received from a user, the message or file content is made available to the multi-agent system, thus it can create a delegated agent that carries the particular characteristics of each system user. This agent is identified using the Media Access Control (MAC) address of the device used to communicate user's data with the server. On behalf of users, agents start to interact, cooperate and negotiation with other system actors in order to achieve the predefined objectives in the given time frame. These objectives are related to particular bartering 


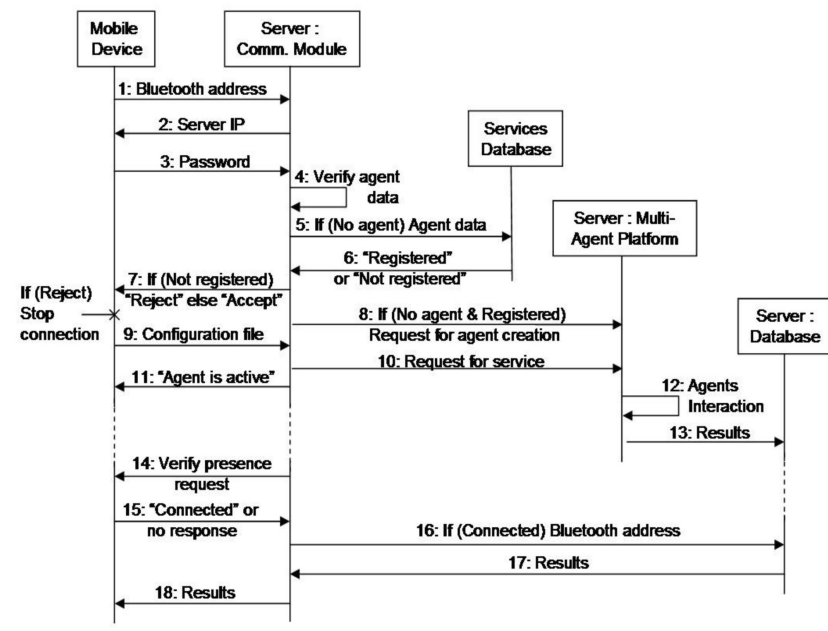

Fig. 2. The Service Accessibility Procedure of ANDIAMO

services, which make them complicated and hardly realized in real life scenario without involving sophisticated technology.

Among other benefits, JACK was chosen to handle all of the agent's interactions because of its ability to meet the requirements of large dynamic environments, which allow programmers of agents to enrich their implementations with the possibility to compatibly access several of the system resources. JACK has also made the communication language applied among involved agents with no restrictions, which made any high-level communication protocol such as KQML [3] or FIPA ACL easily accepted by the running architecture.

\section{ANDIAMO PLATFORM DESCRIPTION}

Andiamo, our carpooling application is written in Java and uses JSR-82 and JSR-120 [4] which are the Bluetooth and the Wireless Messaging API for Java. The application starts a continuous search for Bluetooth-enabled devices and whenever it finds a server, the software on the device establishes a connection with the server and sends the requests related to the Rideshare services. The request is then processed by the server and the results are sent back to the user

Fig. 2 depicts the service accessibility procedure of ANDIAMO. A specific communication module on the server is responsible for managing the interaction with the mobile device. This module receives the Bluetooth address and the password from the mobile device (steps 1 and 3) and checks in the platform running on the server whether a Personal Agent (PA) is assigned to that mobile device (step 4). The module employs the Bluetooth address and the password to map the mobile device with a specific PA. If there is no PA previously assigned to this user, the communication module connects to the central services database and verifies whether the user is registered to the system (steps 5-6). In case of a positive response, it creates a new agent and assigns it to the mobile device user (step 8). The mobile device sends the configuration file to the communication module (step 9), which forwards all the user requests to the appropriate PA (step 10).

The PA then starts interacting with other agents on the platform trying to satisfy all the user requests (step 12). In our example a PA receives one or more requests for finding or asking rides. If the agent reaches an agreement with another agent about their users requests it stores the results locally in the server database (step 13). Later the results could be sent back to the user (steps 14-18).

\section{Service Application Wireless Deployment}

While a current user equipment will already have many the following technologies installed. We start this section by putting the Bluetooth technology (i.e., 802.15) and Wi-Fi (i.e, 802.11 family) in one group and UMTS in another group as in Table I from [5].

We can see that the number of users that can interconnect with each other can vary between tree technologies. In our experiments we used limited number of UEs since Bluetooth technology allows only seven active slaves and one master. Hence the information data bank given from such small set of users is limited. However the implementation of new technologies (Wi-Fi or UMTS) allows to increase the number of received requests and respectively to improve the service. The extension of data bank can provide more complex evaluation of each subscriber request and has more resources for replay.

Wi-Fi technology is widespread around the world. The Wireless local area network (WLAN) is easy to install in any environments. WLAN also can be scalable by adding few access points (AP). These APs involve huge number of new users (up to 100 each) that can share information with each other. Each AP can be as a brick of global network for the current people association. The system consists of these APs builds the set of servers. The information bank which can be supported by a set of servers can store more data requests to let a WLAN-based system with new protocols serve a wider range audience. Such kind of configuration can be distributed in close community environments where an access to the inner network controlled and provided by security system.

However, public wireless networks become very popular in Europe. These networks are broaden by local government and also can include shown services provided by applications such BarterCell and ANDIAMO. In our current work we have implemented Bluetooth technology that allows only limited user number to be actively connected to server and interchange information. At the same time user has to be in area of AP coverage which is no more then $10-20 \mathrm{~m}$. In case of Wi-Fi configuration it permits a user to communicate from different areas but from the same community.

Here we present an interconnection system between two different networks which are included in the same university communication system. That is any student has an access to resource of Central Services (CS). These CS are storage of information from all registered user which would like to share their data with others. Noted that all students at university have a security password to enter to the system hence each of 
TABLE I

ANALYSIS FOR WI-FI, BLUETOOTH AND UMTS

\begin{tabular}{|c|c|c|c|c|c|}
\hline Parameters & Number of users & Maximum rate & Coverage & Mobility & Frequency \\
\hline Wi-Fi 802.11g & Up to 100 & $54 \mathrm{Mbps}$ & $150 \mathrm{~m}$ & Low & $2400 \mathrm{MHz}$ \\
\hline Bluetooth 802.15(Class 3) & Up to 7 & $\begin{array}{l}\text { Asymmetric: } 723,2 \mathrm{kbps} / 57.5 \mathrm{kbps} \\
\text { Symmetric: } 433.9 \mathrm{kbps}\end{array}$ & $10 \mathrm{~m}$ & Low & $2400-2483.5 \mathrm{MHz}$ \\
\hline UMTS & Cell capability & Up to $14.0 \mathrm{Mbps}$ & Global & High & $\begin{array}{c}\text { Uplink:1885-2025 MHz } \\
\text { Downlink:2110-2200 MHz }\end{array}$ \\
\hline
\end{tabular}

them can easily connect to network and ask about BarterCell service. In case if he/she does not have the required application the network makes possible downloading it from CS by agent request from user. It is not necessary that both networks are close to each other. They can be placed in different parts of city but the connection between them is established by Wireless Metropolitan Area Network (WMAN), for example WiMAX. This scheme promises to increase the number of user and also the Data Base of services.

On the other hand any WLAN has security system that does not allow user from outside connect to data server and hence offer his services or ask about any services. Moreover UMTS allows mobile UE change his/her location, and service can follow by him/her. Because of it we can consider UMTS as a friendlier network for strangers which would like to share interests. Also UMTS can provide set of packet-data based services:

- Conventional Internet services

$$
\begin{aligned}
& \text { - Web-browsing } \\
& \text { - electronic mail } \\
& \text { - file transfer } \\
& \text { - video/audio streaming } \\
& \text { - e-commerce, ... }
\end{aligned}
$$

- Combination with location information and mobility

$$
\text { - Location based services, }
$$$$
\text { - navigation }
$$

Exactly based on these benefits of UMTS we can consider our two applications in the global network environment. One of the possible scenarios of ANDIAMO integration could be by means of the cellular technology. A mobile user (NodeB1) can send to the network a request about a service. The network inquires of the Server/DB about user's information in sense of to verify his/her profile or create a new one.

Here, we can propose two ways of development for an integration of our application platforms ubiquitous. First, it can be the local environment in the level of universities, student's campuses, office buildings, and fabric areas. In this contest we can apply WLAN as a base for the deployment of data interchanging. But in this case only registered customers can use information and "talk" by agent intercommunication. At the same time APs can be connected to outside hot spots which will be able to provide information from other networks. Second scenario is to integrate applications to cellular phone in manufactory level and provide services in global network environment such UMTS or allows user to download this application immediately from network, install, and run it by system itself. But path includes new problems and development directions which presented in next section.

\section{CONClusions \& Future WORK}

We investigated the connectivity enhancement of two different agent-based mobile service applications - BarterCell \& ANDIAMO - that are deployed in closed virtual environment such as an enterprise or a university campus. The newly considered communication protocols for BarterCell application can be applied to interconnect different User Equipment (UE). That will also support robust file exchange between nodes. In case of ANDIAMO, the application will allow software agents to interchange and move freely within a range of networks, which will consequently affect the speed of negotiation among agents that are resolving a complex task.

In our future work we intend to exploit one of the emerging technologies such as Software Defined Radio (SDR)[6]. SDR allows a user to download, install, and run software without efforts from user side. Moreover controlling and monitoring of procedures can be done by agent applications. These software agents can later present a user in the network and make all jobs with required parameter by themselves. At the end user will see just friendly interface with replay and already installed and run indispensable software.

\section{ACKNOWLEDGMENT}

Authors would like to acknowledge the support of the Science Foundation Ireland (SFI) under the grant 07/CE/I1147 as well as the European regional Development Fund (ERDF) for supporting partially the SC project trough the Ireland Wales Program (INTERREG 4A). Authors would like to thank Stefano Fante, Oleksiy Chayka, Francesco Sottini and Paolo Giorgini for the effort they have made in implementing ANDIAMO and BarterCell.

\section{REFERENCES}

[1] Abdel-Naby, Sameh. Fante, Stefano and Giorgini, Paolo. Auctions Negotiation for Mobile Rideshare Service. In the Proceeding of the IEEE Second International Conference on Pervasive Computing and Applications (ICPCA07), July 26-27, 2007, Birmingham, UK.

[2] M. Clark. JACKTM Intelligent Agents: An Industrial Strength Platform, chapter 7, pages 175-193. In Multi-Agent Programming, ed. Rafael H. Bordini, 2005.

[3] T. Finin, R. Fritzson, and D. McKay. A language and protocol to support intelligent agent interoperability. In The Proceedings of the CE\&CALS Conference, Washington, USA, June 1992.

[4] http://www.jcp.org/en/jsr/detail?id=82

[5] Marcus Taylor. Understanding Wap : Wireless Applications, Devices, and Services. Artech House Telecommunications Library. June 2000.

[6] J. Mitola III, Software Radio Architecture: Object-Oriented Approaches to Wireless Systems, (new ed.). Wiley, New York: 2004. 